with outcomes such as depression and wellbeing. However, its relationship with objective cognitive performance in older adulthood has been less explored. In this online daily diary study, we examined the daily relationships between awareness of age-related losses and cognitive performance. On eight consecutive days, 116 older adults (aged 60-90) reported AARC losses and completed three cognitive tasks via Qualtrics software. Days when participants perceived more age-related losses were associated with lower performance on inductive reasoning (i.e., letter series task) compared to days with fewer perceived losses. This potentially detrimental effect of AARC losses was not found for episodic memory and perceptual speed across days. Different findings across tests speak to the importance of domain specificity for studying cognitive aging as well as its relationship with subjective perceptions and beliefs about aging.

\section{DAILY WORRIES ABOUT GROWN CHILDREN AND AGING PARENTS AND SLEEP}

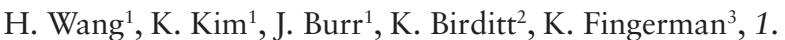
University of Massachusetts Boston, 2. The University of Michigan, 3. The University of Texas at Austin

Sleep is a fundamental health behavior that has profound implications of individual's wellbeing. Though worry has been recognized as one major cause of sleep problems, it is still unclear how middle-aged adults' worries toward adult children and aging parents influence, and influenced by sleep in everyday life. This study examined the association between worries about family members (i.e., grown children and aging parents) and sleep on a daily basis. Middle-aged adults $(\mathrm{N}=191)$ from the second wave of the Family Exchange Study reported on worries about each of their grown children $(\mathrm{n}=454)$ and aging parents $(\mathrm{n}=253)$ as well as sleep duration and sleep quality for seven days (1,261 days). Results from multilevel models showed that same day worries about aging parents and prior day worries about grown children were associated with poorer sleep quality. Further, middle-aged adults were less likely to worry about grown children when they slept longer the previous night, and less likely to worry about aging parents after reporting a good night's sleep. Findings suggested a bidirectional association between worries about family members and sleep. Pre-sleep worries about family members appear to lead to poorer sleep quality at night; poor sleep quality then increases the risk of worrying about family members on the subsequent day. Our findings have implications for health professionals for promoting sleep health in multigenerational families.

\section{DEATH-TALK MAY PREDICT DEATH ACCEPTANCE AMONG ADVANCED CANCER PATIENTS \& SPOUSE CAREGIVERS}

S. Bybee ${ }^{1}$, L. Ellington ${ }^{1}$, K. Cloyes ${ }^{2}$, J. Billitteri ${ }^{3}$, W. Hull' , E. Iacob ${ }^{3}$, M. Reblin ${ }^{4}$, M. Clayton ${ }^{3}, 1$. University of Utah, 2. Univeristy of Utah College of Nursing, 3. University of Utah College of Nursing, 4. Moffitt Cancer Center

Advanced cancer patients on hospice and their spouse caregivers are encouraged to talk about and make plans for the end of life. In previous communication research both pronouns and emotion terms have been examined to assess the presence and degree of personal agency and collaborative coping. Recent studies also suggest that both patient and spouse caregiver death-talk (the use of death-related words such as "dead", "die", "dying", "death") reflects greater death acceptance among patients and caregivers. This secondary data-analysis of a multi-site observational study analyzed audio-recorded home visits of 76 hospice cancer patient-spouse dyads. Conversation segments were analyzed using the Linguistic Inquiry and Word Count (LIWC) program and descriptive statistics, to compare frequency of death-talk in relationship to death acceptance between patients and their caregivers. Most caregivers were female $(60.5 \%)$, slightly younger than patients $(M=65.2$ vs. 67.7 years) and the average length of relationship was 35.4 years. While modest, overall, patients used proportionately more death-talk than spouse caregivers $(.06 \%$ vs $.04 \%$ ) supporting the idea that patients are more willing to use these terms. Following this preliminary analysis, further exploration will illuminate the use of death-talk and its association with death acceptance and relationship quality. The findings from this study will lead to better understanding how using death-talk may reflect the psychological state of death awareness and death acceptance, both in the patient and caregiver, as well as within their relationship. This new information may further the development of interventions supporting advanced cancer patients and spouse-caregivers during this difficult end-of-life period.

\section{DECLINES IN MOTOR TRANSFER FOLLOWING UPPER EXTREMITY TASK-SPECIFIC TRAINING IN OLDER ADULTS}

C. Walter ${ }^{1}$, C. Hengge ${ }^{2}$, B. Lindauer ${ }^{2}$, S. Schaefer ${ }^{3}, 1$. University of Arkansas for Medical Sciences, 2. University of Utah, 3. Arizona State University

Age-related declines in function can limit older adults' independence with activities of daily living (ADLs). While task-specific training maybe a viable approach to improve function, limited clinical resources prevent training on all possible tasks. Thus, training on one task for the benefit of another (i.e., transfer) is important in geriatric physical rehabilitation. The purpose of this study was to test whether motor transfer would occur between two functionally different tasks that simulate ADLs in a sample of older adults. Ninety community dwelling adults ages 43 to 94 years old performed a functional dexterity and functional reaching task at baseline, and were then assigned to one of two groups. The training group completed three days of taskspecific training on the functional reaching task, whereas the no-training group received no training on either task. Groups were re-tested on both tasks on Day 3. No significant interactions were observed between group (training vs. no-training) and time (baseline vs. re-test) on the functional dexterity task (i.e. transfer task), indicating no transfer. However, post hoc bivariate linear regression revealed that, within the training group, the amount of transfer was inversely related to age, such that younger participants showed more transfer than older participants $(\mathrm{r}=-0.42 ; \mathrm{p}=0.0064)$. There was no significant relationship between age and motor transfer for the no-training group $(\mathrm{r}=0.05 ; \mathrm{p}=0.73)$. Our results suggest that motor transfer was both experience- and age-dependent, such that motor transfer may decline with advanced age. Future research will consider how cognitive aging influences transfer of motor skills across ADLs. 